\title{
DIRECT NUMERICAL SIMULATION OF TURBULENT CHANNEL FLOW WITH SPANWISE ALTERNATIVELY DISTRIBUTED STRIPS CONTROL
}

\author{
WEIDAN NI ${ }^{1,2}$, LIPENG LU ${ }^{1}$, JIAN FANG ${ }^{1,2, *}$, CHARLES MOULINEC ${ }^{2}$ AND YUFENG YAO ${ }^{3}$ \\ ${ }^{1}$ National Key Laboratory of Science and Technology on Aero-Engine Aero-Thermodynamics, School of \\ Energy and Power Engineering, Beihang University, 37 Xueyuan Road, Haidian District, \\ Beijing, 100191, China \\ ${ }^{2}$ Scientific Computing Department, Science \& Technology Facilities Council Daresbury Laboratory, Keckwick \\ Lane, Daresbury, Warrington, WA4 4AD, United Kingdom \\ ${ }^{3}$ Department of Engineering Design and Mathematics, University of the West of England, Frenchay Campus, \\ Coldharbour Lane, Bristol, BS16 1QY, United Kingdom \\ *Correspondence: jian.fang@stfc.ac.uk \\ Received Day Month Day \\ Revised Day Month Day
}

\begin{abstract}
The effect of spanwise alternatively distributed strips (SADS) control on turbulent flow in a plane channel has been studied by direct numerical simulations to investigate the characteristics of largescale streamwise vortices (LSSVs) induced by small-scale active wall actuations, and their potential in suppressing flow separation. SADS control is realized by alternatively arranging out-of-phase control (OPC) and in-phase control (IPC) wall actuations on the lower channel wall surface, in the spanwise direction. It is found that the coherent structures are suppressed or enhanced alternatively by OPC or IPC respectively, leading to the formation of a vertical shear layer, which is responsible for the LSSVs' presence. Large-scale low-speed region can also be observed above the OPC strips, which resemble large-scale low-speed streaks. LSSVs are found to be in a statistically-converged steady state and their cores are located between two neighbouring OPC and IPC strips. Their motions contribute significantly to the momentum transport in the wall-normal and spanwise directions, demonstrating their potential ability to suppress flow separation.
\end{abstract}

Keywords: SADS Control; OPC/IPC; Channel Flow, DNS.

\section{Introduction}

Boundary layer separation is one of the most significant flow problems encountered in fluid engineering applications. One of the main issues related to boundary layer separation is that it increases drag force. Passive vortex generator (VG) [1] is a common pathway to reduce drag by enhancing momentum transport of near-wall fluids. While this concept works well, it faces certain technical difficulties in practical applications such as design integration and manufacturing because of the required local shape change. Besides vortex generators, it is also worthwhile citing other methods that can generate large-scale flow structures by activating near-wall small-scale turbulence, such as alternatively distributed riblets-based [2] or wall roughness-based [3] methods. In this paper, spanwise alternatively distributed strips (SADS) control, made of alternatively imposed out-of- 
phase control (OPC) and in-phase control (IPC) wall-normal velocity actuations, are applied to a turbulent plane channel flow. The study mainly focuses on the generation of large-scale streamwise vortices (LSSVs) by SADS in order to suppress flow separation.

\section{Methodology}

The three-dimensional unsteady compressible Navier-Stokes equations are solved using direct numerical simulation (DNS). The flow and computation conditions are set as: $R e=$ 6,000 (based on the bulk velocity $u_{\text {bulk }}$ and the half height of the channel $h$ ), $M=0.2, P r$ $=0.72$; the size of the computational domain is $2 \pi h \times 2 h \times \pi h$; the number of nodes is $392 \times 192 \times 256$. The DNS methodology, numerical scheme and code validation for both uncontrolled and controlled channel flows can be found in Ref. 4. The topography configuration of the controlled case Nstrip4 is presented in Fig. 1. The upper channel wall is kept as a smooth non-slip wall whereas the lower channel wall undergoes alternated OPC and IPC active wall-normal velocity actuations in the spanwise direction, given by $v_{w}(x, z)=\mp A_{O P C} v\left(x, y_{d t c}, z\right)$ respectively. $v_{w}(x, z)$ is the wall-normal velocity at the lower wall and $y_{d t c}$ is the non-dimensionalized fixed distance from the wall, estimated at a value in wall unit of $y_{d t c}^{+} \approx 11$. The coefficients $A_{O P C}$ and $A_{I P C}$ control the amplitude of the wall-normal velocities and are set to be 0.5 in the present study to keep the simulations stable over large computational time. The other two velocity components at the wall are kept to zero. An unperturbed plane channel flow (Case NC) is conducted first as the baseline case.
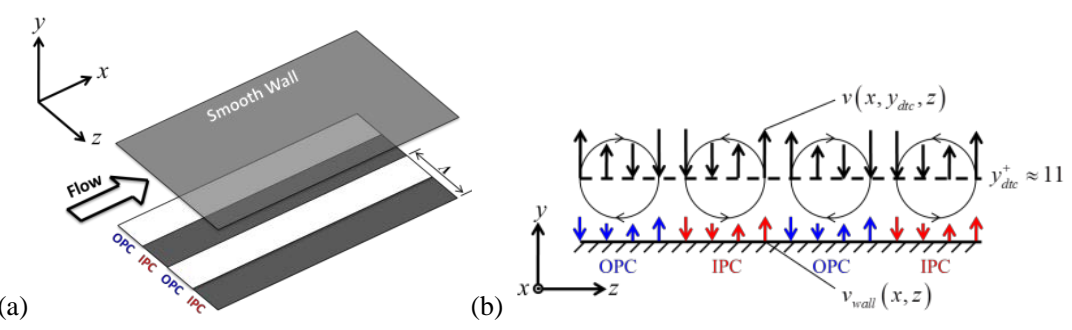

Fig. 1 Topography configuration of Case Nstrip4 (a) and principle of the control method (b).

\section{Results and Discussion}

First, the turbulent coherent structures developed in the lower half of the channel are visualised using the swirling strength $\lambda_{c i}$ iso-surfaces, coloured by the instantaneous streamwise velocity $u$ as indicated in Fig. 2. It is found that the turbulent coherent structures are enhanced above the IPC strips but suppressed above the OPC strips. Further, the mean flow field

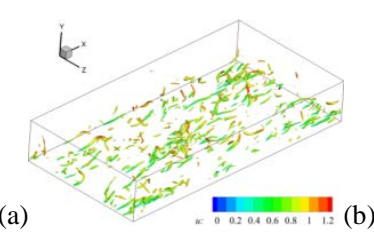

(b)

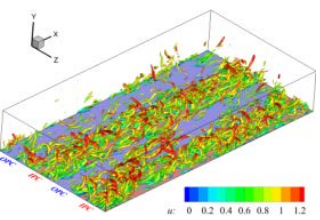
statistics are presented with the Fig. 2 Turbulent coherent structures. (a) Case NC; (b) Case Nstrip4. 
mean skin friction coefficient $C_{f}$ computed by,

$$
C_{f}(z)=\frac{\mu_{w} \partial\langle\bar{u}\rangle_{x} /\left.\partial y\right|_{w}}{0.5 \rho_{b u l k} u_{b u l k}^{2}} .
$$

Here, ${ }^{-}$stands for the time-averaging operator and $\langle\quad\rangle$ for the space-averaging operator. It can be seen from Fig. 3(a) that the skin friction coefficient is slightly reduced over the OPC strips, whereas it rises to a large extent over the IPC strips possibly due to the increase in local turbulence intensity. This is consistent with the coherent structures observed in Fig. 2. The substantial spanwise skin friction reorganisation induces a vertical shear layer between the OPC and IPC strips, resulting in an increase in momentum transport along the spanwise direction. The spanwise skin friction gradient $\mathrm{d} C_{f}(z) / \mathrm{d} z$ is calculated to illustrate the effect of SADS control on the strength of the spanwise shear-layer (see Fig. 3(b)).

(a)

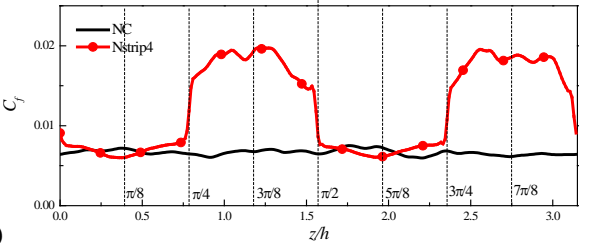

(b)

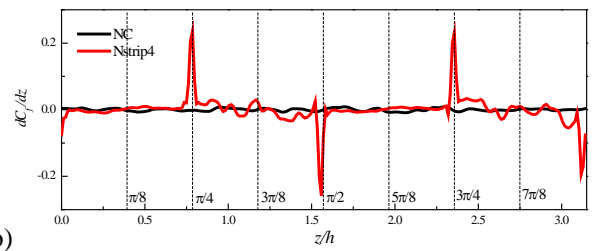

Fig. 3 Mean skin friction coefficient $C_{f}$ (a) and its spanwise gradient (b) based on $x$ - and time-averaging.

Fig. 4 compares the contours of the mean streamwise velocity component $\langle\bar{u}\rangle_{x}$ in the $y-z$ plane, showing the influence of the SADS control on the distribution of the mean streamwise velocity component in the spanwise direction. It is clear that low-speed regions are induced above the OPC strips, which resemble large-scale low-speed streaks. In contrast, the near-wall velocity increases above the IPC strips. The simulated spanwise distribution of the local mean velocity is qualitatively in good agreement with the experimental observations of Nugroho et al. [2] and Mejia-Alvarez et al. [5].

(a)

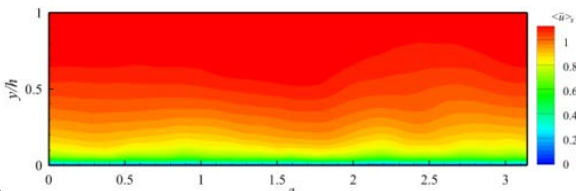

(b)

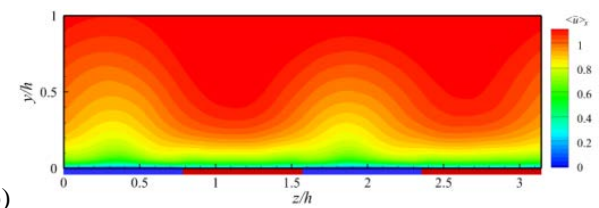

Fig. 4 Distribution of the $x$ - and time-averaged streamwise velocity component. (a) Case NC; (b) Case Nstrip4. Contours of the mean swirling strength $\Lambda_{c i}$ (see Ref. 6) of Case Nstrip4 are presented in Fig. 5(b) and compared to that for Case NC in Fig. 5(a). For Case NC, some small-scale negative and positive $\Lambda_{c i}$ regions are randomly distributed. For Case Nstrip4, a clear distribution of $\Lambda_{c i}$ is identified. Large-scale negative (resp. positive) $\Lambda_{c i}$ clouds are located at the left (resp. right) hand sides of the OPC strips. This indicates that the largescale steady counter-rotating streamwise vortices are induced and further sustained by this control method. They enhance the momentum transport throughout the channel. The low-speed fluid is transported from the near-wall region to the central part of the channel 
as shown in Fig. 4(b). Similar large-scale motions were reported by Barros et al. [6] for a flow with irregular transverse roughness and Willingham et al. [3] for a flow with highorder transverse roughness. It can be concluded from Fig. 5 that the large-scale statistically steady counter-rotating streamwise vortices, whose cores are located between the OPC and IPC strips, are generated by SADS control.

(a)

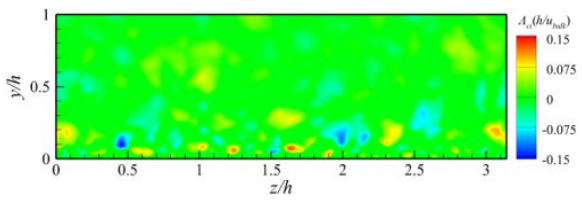

(b)

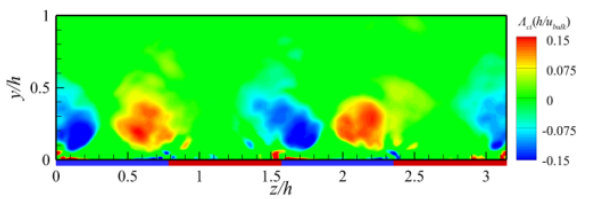

Fig. 5 Distribution of the swirling strength $\Lambda_{c i}$ normalized by $\left(u_{b u l k} / h\right)$ for Cases NC (a) and Nstrip4 (b). The swirling strength depends on the sign of the streamwise vortex $\omega_{x}$.

\section{Conclusions}

The following conclusions are derived from the present research:

- Spanwise alternatively distributed OPC/IPC strips have an impact on the flow field, especially in the spanwise direction. The turbulence coherent structures can be suppressed or enhanced alternatively by the OPC or IPC strips.

- The skin friction decreases and increases alternatively in the spanwise direction. A vertical shear layer is induced at the interface between the OPC and IPC strips. The mean velocity deaccelerates or accelerates alternatively along the spanwise direction.

- The analysis shows that the generated LSSVs have a statistically-converged steady flow motion whose centers are located at the interfaces between the OPC and IPC strips. These motions contribute significantly to the momentum transport in the wall normal and spanwise directions indicating potential for flow separation suppression.

\section{Acknowledgments}

The project is supported by the Key Subjects of the National Natural Science Foundation of China (51420105008, 11572025 and 51476004), the UKTC (EP/L000261/1), and the Department of Research and Advanced Engineering of PSA. The authors also would like to thank EPSRC for using ARCHER and the Hartree Centre for using their machines.

\section{References}

1. J. C. Lin, Prog. Aerosp. Sci., 38 (2002) 389.

2. B. Nugroho, N. Hutchins and J. P. Monty, Int. J. Heat Fluid Fl. 41 (2013) 90.

3. D. Willingham, W. Anderson, K. T. Christensen and J. M. Barros, Phys. Fluids 26 (2014) 25.

4. W. Ni, L. Lu, J. Fang, C. Moulinec and Y. Yao, Flow Turbul. Combust. (In press).

5. R. Mejia-Alvarez and K. T. Christensen, Phys. Fluids 25 (2013) 115109.

6. J. M. Barros, K.T. Christensen, J. Fluid Mech. 748 (2014) R1. 